\title{
MANUFATURA ADITIVA: REVISÃo SISTEMÁTICA DA LITERATURA
}

\author{
Pedro Coelho Silva ${ }^{(1)}$ (ocoelhopedro@gmail.com), Rafael Souza Santandrea ${ }^{(2)}$ \\ (r.santandrea97@gmail.com), Marcos Vinício Antônio Xavier ${ }^{(3)}$ (marcosxavier_1992@hotmail.com), \\ Lincoln Cardoso Brandão ${ }^{(4)}$ (lincoln@ufsj.edu.br) \\ ${ }^{(1)}$ Universidade Federal de São João del-Rei (UFSJ) - Engenharia Mecânica - Praça Frei Orlando, nº 170 - Centro - São João \\ del-Rei-MG \\ ${ }^{(2)}$ Universidade Federal de São João del-Rei (UFSJ) - Engenharia Mecânica - Praça Frei Orlando, nº 170 - Centro - São João \\ del-Rei-MG \\ ${ }^{(3)}$ Universidade Federal de São João del-Rei (UFSJ) - Engenharia Mecânica - Praça Frei Orlando, nº 170 - Centro - São João \\ del-Rei-MG \\ ${ }^{(4)}$ Universidade Federal de São João del-Rei (UFSJ) - Engenharia Mecânica - Praça Frei Orlando, nº 170 - Centro - São João \\ del-Rei-MG
}

RESUMO: A manufatura aditiva é percebida atualmente como um tipo de tecnologia com a tendência de ser uma das principais metodologias de fabricação para o futuro, ainda com crescimento em potencial, mesmo já existindo há mais de 30 anos. Caracterizada pelos mecanismos de fabricação pela sobreposição de camadas, através de diversos mecanismos de união, a manufatura aditiva permite construir geometrias impossiveis ou muito dificeis de se conseguir com outras formas de fabricação. Na última década houve um claro aumento na precisão dos equipamentos, redução dos custos, maior variedade de materiais aplicáveis e possiveis aplicações. Através da revisão sistemática da literatura, pesquisando artigos que seguem o mesmo tipo de análise, a presente investigação objetiva identificar quais fatores permitiram sua popularização recente e quais dificultaram sua implementação anteriormente. Foram elencadas a extinção das principais patentes, permitindo o início de pesquisas sem necessidade do licenciamento e, com essas, o início de uma nova classe de equipamento, de menor custo, ampliando também o alcance possível do mercado.

PALAVRAS-CHAVE: Manufatura aditiva, fabricação aditiva, impressão 3D, estado da arte, revisão sistemática.

\section{INTRODUÇÃO}

Apesar de existir há mais de 30 anos, a Manufatura Aditiva (AM, sigla do inglês para Additive Manufacturing), pesquisada na década de 70, foi registrada somente a partir de 1986 na primeira patente de Estereolitografia. Com a capacidade de gerar sólidos de dimensões próximas do objetivo em um processo único, era considerada uma metodologia da Prototipagem Rápida (RP, sigla do inglês para Rapid Prototyping), e a Impressão Tridimensional (3DP, sigla do inglês para $3 D$ Printing) era uma dos processos possíveis (CHUA; CHOU \& WONG, 1998; LEVY; SCHINDEL \& KRUTH, 2003; DIMITROV; SCHEVE \& DE BEER, 2006; WONG \& HERNANDEZ, 2012; ROSOCHOWSKI \& MATUSZAK, 2000) porém observa-se a tendência do desuso dos termos RP (RAPID, 2019) e Manufatura (MANUFACTURING, 2019), com a tendência de aumento da procura por AM (ADDITIVE, 2019) e 3DP (3D, 2019) desde 2009. 

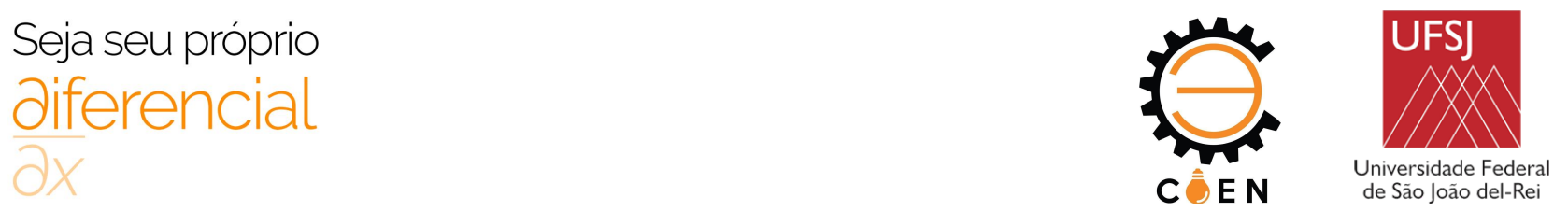

A AM é definida como os processos de fabricação com o objetivo de se criar um objeto tridimensional por camadas a partir de um modelo virtual. Apenas nos últimos cinco anos foi normatizada pela série ASTM 52900 e as normas ISO 17296, DIS 20195 e ASTM F2792 (CULMONE; SMIT \& BREEDVELD, 2019; GARDAN, 2019; KOESTER et al., 2016; JAFFAR-BANDJEE; CASAS \& KRIJNEN, 2018; MA et al., 2018), sendo subdividida em sete categorias disponíveis comercialmente: jateamento de aglomerante; deposição direta por aplicação de energia; extrusão de material; jateamento de material; fusão seletiva de material em pó; laminação de chapas e fotopolimerização de líquido (CULMONE; SMIT \& BREEDVELD, 2019, tradução nossa).

Na última década, com a evolução da tecnologia, a AM se estende a novos mercados por permitir a obtenção de geometrias complexas e uma maior variedade de materiais (VAYRE et al., 2012; KAPNER et al., 2018; STEWART, 2019), se destacou dentre as demais metodologias de fabricação para protótipos e apresenta o potencial para a fabricação de ferramentas (ROSOCHOWSKI \& MATUSZAK, 2000), produtos finais (NIAKI, 2019) e arte (BICKEL et al., 2018).

\section{OBJETIVO}

A presente investigação tem o objetivo de identificar os fatores cruciais que permitiram a popularização da tecnologia de manufatura aditiva na última década.

\section{METODOLOGIA}

Pesquisas de caráter bibliográfico com o objetivo de mapear, delimitar e discutir um tema, em caráter inventariante e descritivo, são definidas como "estado da arte" ou "estado do conhecimento" segundo Ferreira (2002), ou "revisão sistemática" segundo Sampaio e Mancini (2007). Assim deve-se ser metódico, explícito e permitir a reprodução, de forma a nortear o desenvolvimento de projetos e futuras investigações, por identificar as tendências tecnológicas e metodológicas mais aplicadas e seus potenciais (SAMPAIO \& MANCINI 2007).

Focado nas inovações em fabricação, a presente investigação buscou artigos recentes sobre o tema e destacou-se a impressão tridimensional como termo em tendência elevada em pesquisas dentre os tipos de fabricação (MANUFACTURING, 2019). Para verificar a reprodutibilidade da metodologia, serão analisadas pesquisas que aplicaram o mesmo tipo em relação aos termos considerados sinônimos da impressão tridimensional e termos complementares, em inglês, por demonstrarem maior número de referências, verificando também sua evolução.

As publicações serão classificadas e comparadas entre si quanto às categorias e materiais analisados e ao ano de lançamento, e analisados em relação à tendência da popularidade do assunto, demonstrado pela procura sobre os termos (ADDITIVE, 2019; 3D, 2019) e os fatos históricos destacados pelos autores. Também serão verificadas a confirmação das tendências destacadas pelos autores. 


\subsection{Critérios de elegibilidade}

Os artigos foram selecionados à partir das seguintes regras:

1. Conter no título e/ou palavras-chave os termos, traduções ou sinônimos:
a. Impressão Tridimensional;
b. Manufatura Aditiva;
c. Prototipagem Rápida.

2. Selecionar e analisar referências pelas metodologias ou similares:

a. Estado da arte;

b. Estado do Conhecimento;

c. Revisão Sistemática.

3. Analisar, ou comparar no mínimo duas categorias de fabricação de manufatura aditiva.

Foram selecionados vinte e um artigos para serem analisados. Para auxiliar na conclusão foi feita uma nova procura, substituindo a terceira regra para "conter citação de 'inovação aberta', tradução ou sinônimo", acrescentando mais seis artigos.

\section{REVISÃO SISTEMÁTICA DE MANUFATURA ADITIVA}

Os artigos selecionados foram classificados pela revisão da tecnologia segundo a classificação das Manufaturas Aditivas definidas pela norma ASTM 52900-15 - Terminologia padrão para manufatura aditiva (CULMONE; SMIT \& BREEDVELD, 2019, tradução nossa), listadas abaixo.

- Jateamento de aglomerante (BJ, sigla do inglês para Binder Jetting) - fabricação por aplicação seletiva de um líquido aglomerante à um material em pó;

- Deposição direta por aplicação de energia (DED, sigla do inglês para Direct Energy Deposition) - fabricação cujo processo consiste da aplicação de energia térmica concentrada para fundir o material sólido enquanto é depositado;

- Extrusão de material (ME, sigla do inglês para Material Extrusion) - fabricação por extrusão e deposição de um termoplástico ou solução por meio de um bocal, incluindo:

○ Modelamento por deposição de material fundido(FDM, sigla do inglês para fused deposition modeling);

- Jateamento de material (MJ, sigla do inglês para Material Jetting) - fabricação por deposição seletiva de material líquido, curado por energia luminosa à cada camada;

- Fusão seletiva de material em pó (PBF, sigla do inglês para Powder Bad Fusion) fabricação em que a energia térmica funde seletivamente regiões em uma "cama" de pó;

- Laminação de chapas (SL, sigla do inglês para Sheet Lamination) - fabricação por corte, sobreposição e união de chapas de material;

- Fotopolimerização de líquido (VP, sigla do inglês para Vat Photopolymerization) fabricação por polimerização de um líquido pela aplicação de laser ou energia luminosa, incluindo:

- Estereolitografia (STL, sigla do inglês para stereolithography); 

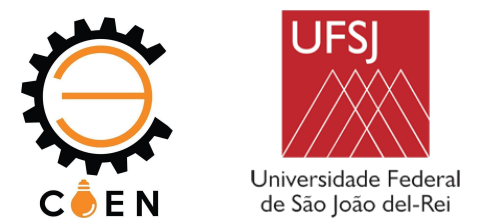

- Processamento Digital de luz (DLP, sigla do inglês para digital light process)(CULMONE; SMIT \& BREEDVELD, 2019, tradução nossa).

Além disso também foram classificados os tipos de materiais das aplicações estudadas, sendo classificados como Cerâmicos, Metálicos, Orgânicos ou Poliméricos, além do ano de publicação e o autor principal.

\subsection{Terminologia}

É importante observar que os artigos anteriores à 2016 divergiam quanto à terminologia utilizada em relação à norma atual. Os artigos priorizavam os termos registrados pelas patentes e demais referências estudadas, como Levy e colaboradores (2003) destacam:

- Estereolitografia

- Solidificação por cura

- Fabricação por laminação

- Modelamento por deposição de material fundido

- Sinterização seletiva a laser

- Impressão tridimensional (gotejamento na "cama” de pó) (LEVY; SCHINDEL \& KRUTH, 2003, tradução nossa).

Destaca-se que ainda nesse artigo, a solidificação por cura (SGC, sigla do inglês para Solid Ground Curing) foi considerada em desuso desde 1999 (LEVY; SCHINDEL \& KRUTH, 2003).

Além disso o nome da própria metodologia de fabricação se diverge, sendo chamada de fabricação em camadas (do inglês Layer Manufacturing), fabricação por forma livre (do inglês freeform fabrication) (CHUA; CHOU \& WONG, 1998; SEARS, 1999; LEVY; SCHINDEL \& KRUTH, 2003; DIMITROV; SCHEVE \& DE BEER, 2006) e em 2006 o termo impressão tridimensional (do inglês, three dimensional printing) foi usada pela primeira vez de forma geral (DIMITROV; SCHEVE \& DE BEER, 2006), sendo o termo mais aplicado nos artigos mais recentes (NIAKI, 2019; KAPNER, 2018; CULMONE; SMIT \& BREEDVELD, 2019; MA, 2018; GARDAN, 2016, 2019; QURENSHI, 2015).

\subsection{Vantagens e desvantagens}

É importante observar que a lista de vantagens e desvantagens publicadas pelos autores variou pouco ao longo dos anos, sendo que as desvantagens listadas por Chua e colaboradores (1998) como a baixa resolução superficial; baixa resistência; limitações de materiais; e a necessidade de múltiplos processos; para alguns tipos de fabricação permanecem (JAFFAR-BANDJEE; CASAS \& KRIJNEN, 2018; KAPNER et al., 2018; Kyle et al., 2017).

Por outro lado as resoluções possíveis foram aprimoradas, saindo de $89 \mu \mathrm{m}$ para a VP e entre 100 e $200 \mu \mathrm{m}$ para BJ (CHUA; CHOU \& WONG, 1998) para 0,5 a $10 \mu \mathrm{m}$ para VP e 80 a 250 $\mu \mathrm{m}$ para $\mathrm{BJ}$, além de maior disponiblidade de materiais disponíveis para todos os processos (JAFFAR-BANDJEE; CASAS \& KRIJNEN, 2018). 


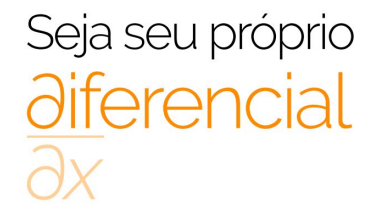

\subsection{Tendências}
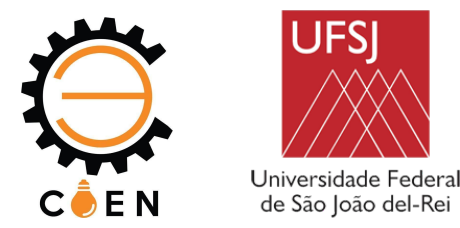

Chua e colaboradores (1998) identificaram que para a maior adoção da tecnologia deveria ser necessário melhorar a precisão e resolução das máquinas, permitindo-se criar peças com melhor resistência física, além de se melhorar as velocidades de produção de forma a tornar concorrente aos métodos tradicionais de fabricação. Rosochowski e Matusk (2000) também destacam o crescente número de pesquisas na área, ampliando o potencial para a descobertas de tecnologias disruptivas, apesar de não ter ocorrido uma mudança de grande importância na tecnologia desde sua invenção. Assim vários autores observaram a evolução da tecnologia, com a aplicação da AM saindo da Prototipagem Rápida, migrando para a ferramentaria rápida (do inglês Rapid Tooling) para os embriões da fabricação rápida (do inglês Rapid Manufacturing), ampliando as possíveis aplicações. Os autores ainda destacam que a falta de informações sobre os equipamentos de fabricação e os produtos; e a baixa variedade de fornecedores e softwares compativeis justificam sua crescente, mas baixa adoção (ROSOCHOWSKI \& MATUSK, 2000; LEVY SCHINDEL \& KRUTH, 2003; DIMITROV; SCHEVE \& DE BEER, 2006).

Pearce e colaboradores (2010) destacam que, graças à maior adoção da internet e a redução do custo computacional, permitiu o aceleramento da evolução das impressoras tridimensionais, cujas patentes principais, geradas na década de 80 , expiraram e permitiram que pesquisas sem fins lucrativos, como a RepRap e a Fab@home, iniciaram o desenvolvimento de equipamentos de baixo custo. RepRap é um projeto iniciado por Adrian Bowyer, na universidade de Bath, com o objetivo de desenvolver equipamentos auto replicantes, ou seja, um equipamento que permite que você construa mais equipamentos iguais à partir do uso do mesmo. As primeiras impressoras foram feitas baseadas em ME. O Fab@home, criado por Hod Lipson e Evan Malone, funcionam de maneira similar, também de baixo custo, porém baseada em MJ. Além disso também destaca a criação de softwares gratuitos e de código livre criados, como o OpenSCAD, reduzindo também o investimento necessário para a operação dos equipamentos. Diferentemente dos pesquisadores anteriores, esses autores destacam que para o desenvolvimento da tecnologia é necessário a redução de custo para os equipamentos e sua operação, que pode ser atingido pelo desenvolvimento aberto dos mesmos (PEARCE et al., 2010). Apesar do destaque aferido por essa pesquisa apenas Sun et al. (2015), Schoffer (2016), Gardan (2016, 2019), Kapner et al. (2018) e Stewart (2019) citam as pesquisas abertas como pontos importantes de inflexão na adoção da tecnologia.

Em consonância com esse destaque, observamos na Figura 1 e Figura 2, as tendências de procura dos termos "manufatura aditiva" e "impressão tridimensional", à partir do Google Trends, que à partir de 2010 houve a tendência acelerada na procura dos termos, após as pesquisas de Bower, Lipton e Malone apresentaram os primeiros protótipos e as referências para se reproduzir os equipamentos criados (PEARCE et al., 2010; 3D, 2019; ADDITIVE, 2019). 


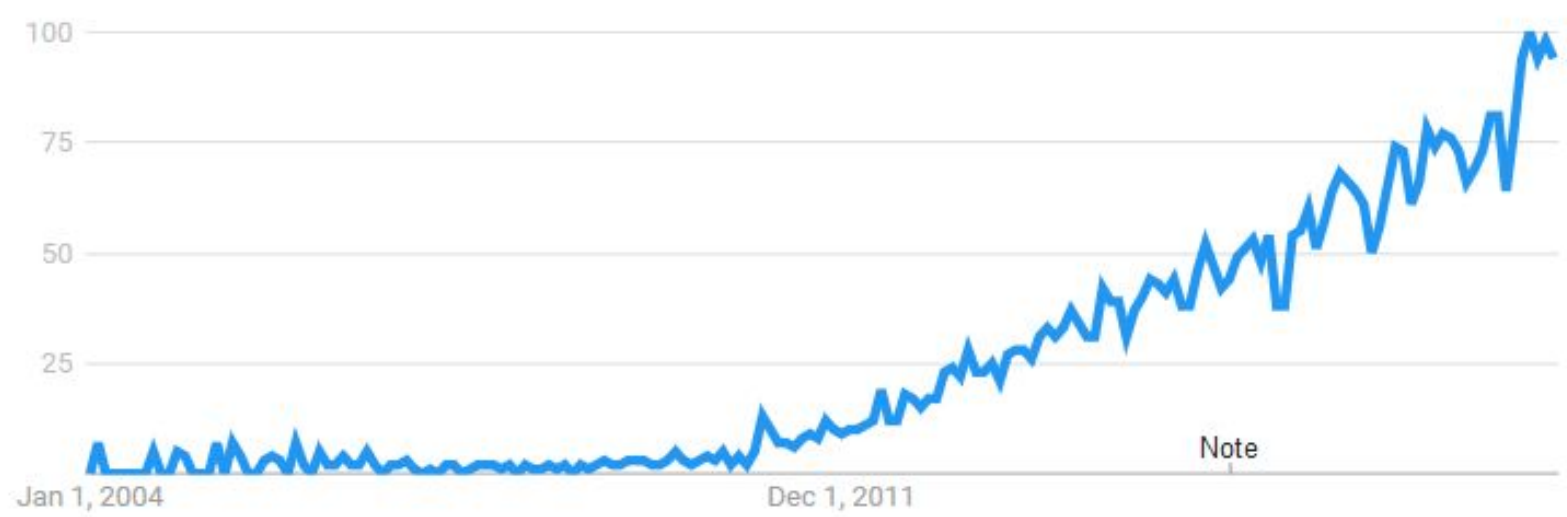

Figura 1. Tendência de busca do termo “Additive Manufacturing”. Fonte: ADDITIVE (2019).

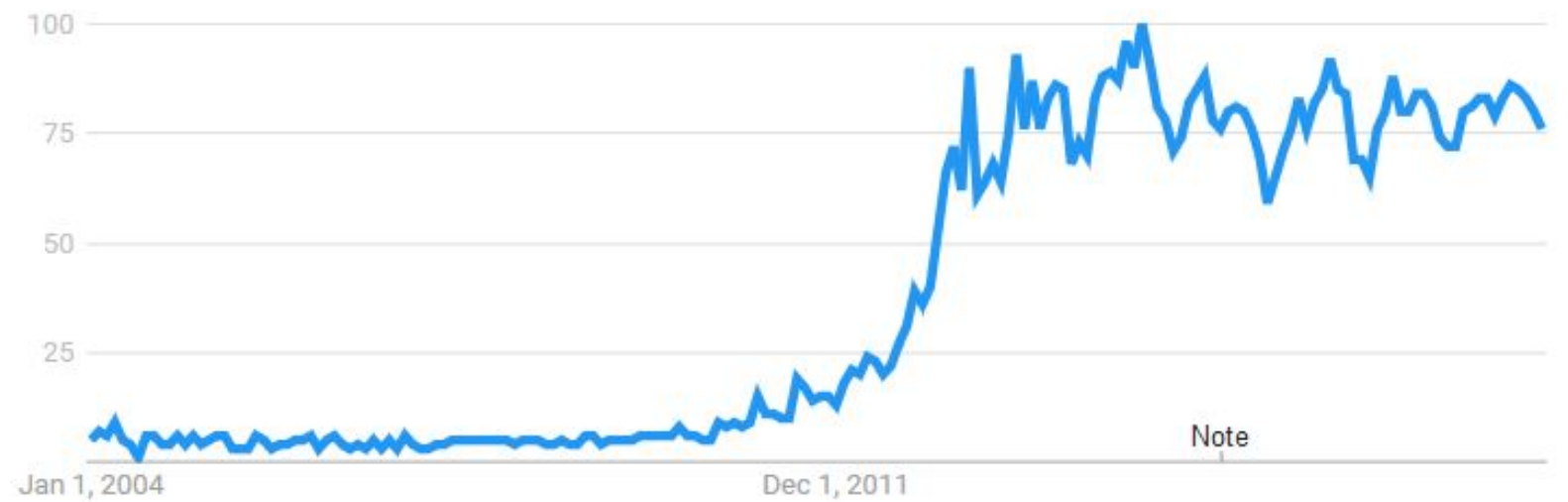

Figura 2. Tendência de busca do termo “3D Printing”. Fonte: 3D (2019).

\subsection{Patentes}

As principais patentes para a metodologia de fabricação aditiva (CHUA; CHOU \& WONG, 1998) são:

- Aparato para produção de objetos tridimensionais por estereolitografia (HULL, 1986, tradução nossa);

- Método e aparato para criar um objeto íntegro por laminação (FEYGIN, 1988, tradução nossa);

- Aparato para modelagem de objetos tridimensionais (CRUMP, 1989, tradução nossa);

- Método e aparato para produzir peças por sinterização seletiva (DECKARD, 1989, tradução nossa);

- Técnicas de impressão tridimensional (SACHS et al., 1993, tradução nossa). 

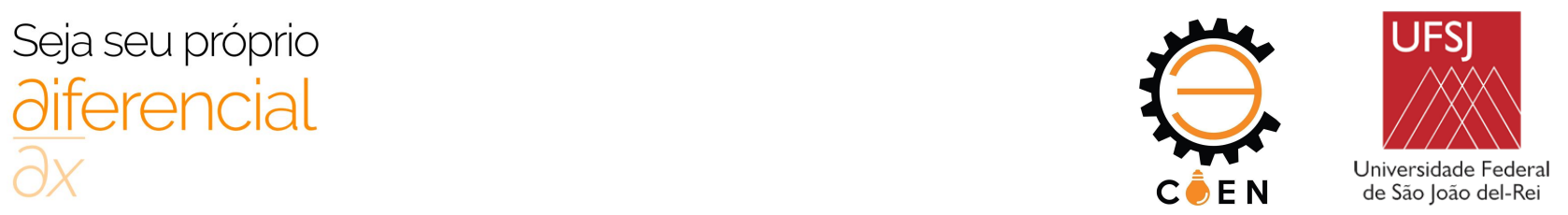

A primeira dessas patentes expirou em 2006 (HULL, 1986) e a última destas patentes expirou em 2011 (SACS, 1993) e, por tanto, à partir desses anos todos os equipamentos produzidos por e para a aplicação desse tipo de tecnologia se vê desempedido de autorização prévia das empresas detentoras dos mesmos. Como consequência, pesquisas como a RepRap e Fab@home iniciaram o desenvolvimento de equipamentos de baixo custo e disponibilizaram para a comunidade online o acesso aos documentos para a reprodução do mesmo (PEARCE et al., 2010).

Ao mesmo tempo, com a popularização da tecnologia, outros pesquisadores registraram mais patentes relacionadas com os termos "Rapid Prototyping", "Additive Manufacturing" e "3D Printing". Podemos observar no repositório Google Patents, que até o ano de 1989 havia aproximadamente cento e cinquenta patentes ; em 1999 havia aproximadamente mil e quinhentas patentes; até 2009 o valor superou oito mil patentes; e atualmente, em 2019, há mais de cinquenta mil patentes. Também é importante observar que até 1989 não havia uma grande concentração das patentes, com o maior detentor com menos de 5\% das mesmas. Em 1999 a 3D System, empresa de Charles Hull (HULL, 1986), destaca-se com mais de 8\%, em 2009, 3,3\% e apenas 1,9\% atualmente. E a Stratys, empresa de Scot C. Crump (CRUMP, 1989), havia 3,6\%, 2,5\% e atualmente possui mais de $11 \%$ das patentes (GOOGLE PATENTS, 2019).

\subsection{Perspectivas futuras}

O mercado de equipamentos de Manufatura Aditiva se vê em crescimento constante. Desde 2010 apresentou um crescimento anual de mais de 4,6\%, chegando à 12,5\% em 2017 em relação ao ano anterior. Segundo estimativas de Stewart (2019) a tendência do mercado é continuar a ascensão acelerada, em valores iguais ou superiores aos de 2017. Kapner e colaboradores (2018), observaram que houve o aumento acelerado na venda de equipamentos de baixo custo, comparado aos de nível industrial, sendo que a primeira classe, com custo de até cinco mil dólares, apresentou crescimento de aproximadamente $100 \%$ ao ano em unidades vendidas até 2014 , a segunda classe apresentou o crescimento de aproximado de $10 \%$ no mesmo período.

Para vários autores, esse crescimento representa o amadurecimento do mercado, como previsto por Levy e colaboradores em 2003. Com a inserção de um produto no mercado ocorre um crescimento acelerado na demanda, seguido por uma desilusão do mercado e consequente redução da demanda. Após esse período o mercado sofre o amadurecimento, com melhor avaliação da tecnologia, desenvolvimento, padronização e por fim a aceitação, com o crescimento da demanda ao longo do processo (LEVY; SCHINDEL \& KRUTH, 2003). Podemos observar que estamos na retomada do crescimento, que estava em um crescimento pequeno até o começo dos anos 2000, houve um crescimento acelerado entre 2009 e 2014, com a introdução dos equipamentos de baixo custo no mercado, e uma desaceleração entre 2015 e 2016. Em 2017, com melhor definição do mercado e a criação das normas específicas para esse tipo de tecnologia, observa-se o crescimento do mercado, focado mais uma vez à indústria (STEWART, 2019).

Além disso, pesquisas na área computacional permitiu a otimização da aplicação da AM, por permitir atingir geometrias impossíveis, ou muito difíceis de se obter por outros métodos, pode-se utilizar as simulações de topologia, definindo à uma peça a geometria com menor massa possível, mas com a mesma resistência de uma peça construída de forma similar (GAYNOR, 2015; GARDAN, 2016, 2019). 

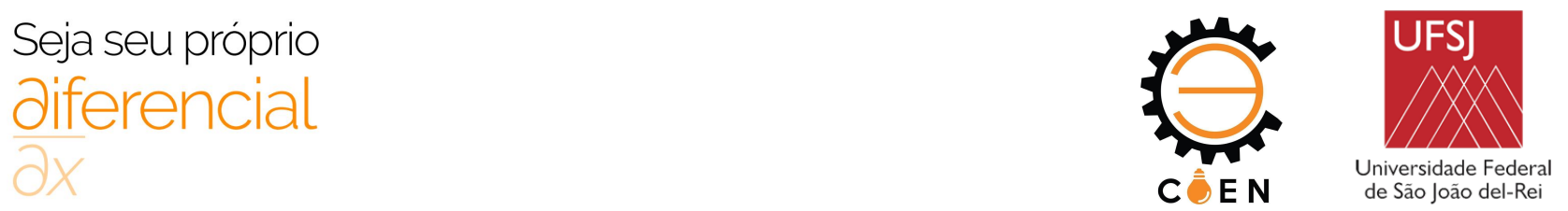

Novos materiais específicos para AM estão sendo desenvolvidos, como compósitos (JAFFAR-BANDJEE; CASAS \& KRIJNEN, 2018; GARDAN, 2016, 2019; KYLE et al., 2017; HAGER; GOLONKA \& PUTANOWICZ, 2016; CULMONE; SMIT \& BREEDVELD., 2019; MA et al., 2018; KOKKINS, 2018; KAPNER et al., 2018; SUN et al., 2015; NIAKI 2019; QURENSHI et al., 2015), biomateriais (JAFFAR-BANDJEE; CASAS \& KRIJNEN, 2018; GARDAN, 2016, 2019; KYLE et al., 2017; HAGER; GOLONKA \& PUTANOWICZ, 2016, CULMONE; SMIT \& BREEDVELD, 2019; MA et al., 2018) e metais especiais (GARDAN, 2016, 2019;CULMONE; SMIT \& BREEDVELD, 2019; MA et al., 2018; KAPNER et al., 2018; NIAKI, 2019).

Além disso, a metodologia está sendo pesquisada e aplicada em níveis diferentes, como equipamentos de alta precisão para medicina em implantes e ferramentas especiais (GARDAN, 2016, 2019; CULMONE; SMIT \& BREEDVELD, 2019; KAPNER et al., 2018; KYLE et al., 2017); e impressoras de grande porte para a construção civil (GARDAN, 2019; HAGER; GOLONKA \& PUTANOWICZ, 2016; BALLETTI; BALLARIN \& GUERRA., 2017; KAPNER et al., 2018; MA et al., 2018).

\section{CONCLUSÃO}

Nos últimos quarenta anos, desde as pesquisas pioneiras em Manufatura Aditiva da década de 70 houve um crescente interesse no assunto. A tecnologia que não teve muito destaque entre alguns dos pioneiros, desde o registro da primeira patente e comercialização, observa-se um crescimento constante da procura literária e do mercado. Há aproximadamente dez anos houve uma revolução no mercado, com o final das patentes principais, houve uma aceleração tanto no mercado quanto nas pesquisas sobre AM e hoje estamos em um mercado em estado de amadurecimento.

A natureza do desenvolvimento de inovação aberta que se deu na última década destaca-se as vantagens de se liberar a aplicação de uma tecnologia, antes restringida por uma patente, que se mostrou vantajosa também para as antigas detentoras das patentes originais, que ainda se destacam na pesquisa comercial.

A tecnologia de AM se mostra com um grande potencial de ampliação, devido à maior variedade de materiais disponíveis; e aprimoramento, pois ainda persistem algumas das desvantagens identificadas há mais de duas décadas.

\section{AGRADECIMENTOS}

Os autores agradecem o apoio da FAPEMIG, do CNPq e da CAPES na concessão de cotas de bolsas e financiamentos para os diversos Programas Institucionais e laboratórios que colaboraram com a presente pesquisa. Agradecem também aos graduandos, auxiliares técnicos e discentes da universidade integrantes dos mesmos.

\section{DIREITOS AUTORAIS}

Os autores são os únicos responsáveis pelo conteúdo das informações contidas neste artigo. 


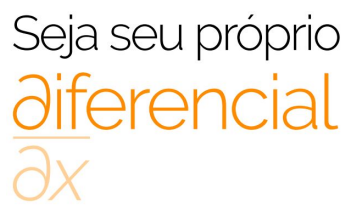

\section{REFERÊNCIAS}
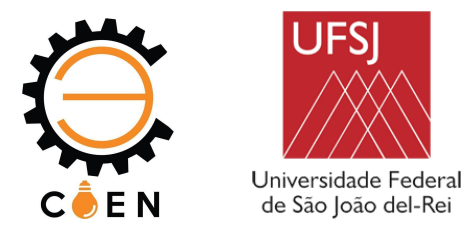

GOOGLE PATENTS seacrh: ((“RAPID PROTOTYPING”) OR ("Additive Manufacturing") OR ("3D Printing")) . Google Patents, 2019. Disponível em: $<$ https://patents.google.com/?q=\%22Rapid+Prototyping\%22,\%22Additive+Manufacturing\%22,\%2 23D+Printing\%22>. Acesso em: 29 de junho de 2019.

3D Printing. Google Trends, 2019. Disponível em: $<$ https://rends.google.com/trends/explore?date=all\&q=3d\%20printing $>$. Acesso em: 29 de junho de 2019.

ADDITIVE Manufacturing. Google Trends, 2019. Disponível em: $<$ https://rends.google.com/trends/explore?date=all\&q=additive + manufacturing $>$. Acesso em: 29 de junho de 2019.

BALLETTI, Caterina; BALLARIN, Martina; GUERRA, Francesco. 3D printing: State of the art and future perspectives. Journal of Cultural Heritage, v. 26, p. 172-182, 2017.

BICKEL, Bernd CIGNONI, Paolo; MALOMO, Luigi; PIETRONI, Nico. State of the art on stylized fabrication. In: Computer Graphics Forum. 2018. p. 325-342.

CHUA, C. K.; CHOU, S. M.; WONG, T. S. A study of the state-of-the-art rapid prototyping technologies. The International Journal of Advanced Manufacturing Technology, v. 14, n. 2, p. 146-152, 1998.

CRUMP, S. Scott. Modeling apparatus for three-dimensional objects. U.S. Patent n. 5,340,433, 23 ago. 1994.

CULMONE, Costanza; SMIT, Gerwin; BREEDVELD, Paul. Additive manufacturing of medical instruments: A state-of-the-art review. Additive Manufacturing, 2019.

DECKARD, Carl R. Method and apparatus for producing parts by selective sintering. U.S. Patent n. 4863538, 5 set. 1989.

DIMITROV, Dimitar; SCHREVE, Kristiaan; DE BEER, Neal. Advances in three dimensional printing-state of the art and future perspectives. Rapid Prototyping Journal, v. 12, n. 3, p. 136-147, 2006.

FEYGIN, Michael. Apparatus and method for forming an integral object from laminations. U.S. Patent n. 4752352, 21 jun. 1988.

FERREIRA, Norma Sandra A. As pesquisas denominadas" estado da arte. Educação \& sociedade, v. 23, 2002.

GARDAN, Julien. Additive manufacturing technologies: state of the art and trends. International Journal of Production Research, v. 54, n. 10, p. 3118-3132, 2016.

GARDAN, Julien. Smart materials in additive manufacturing: state of the art and trends. Virtual and Physical Prototyping, v. 14, n. 1, p. 1-18, 2019.

GAYNOR, Andrew Thomas. Topology optimization algorithms for additive manufacturing. 2015. Tese de Doutorado. Johns Hopkins University.

HAGER, Izabela; GOLONKA, Anna; PUTANOWICZ, Roman. 3D printing of buildings and building components as the future of sustainable construction?. Procedia Engineering, v. 151, p. 292-299, 2016.

HULL, Charles W. Apparatus for production of three-dimensional objects by stereolithography. U.S. Patent n. 4575330, 11 mar. 1986. 

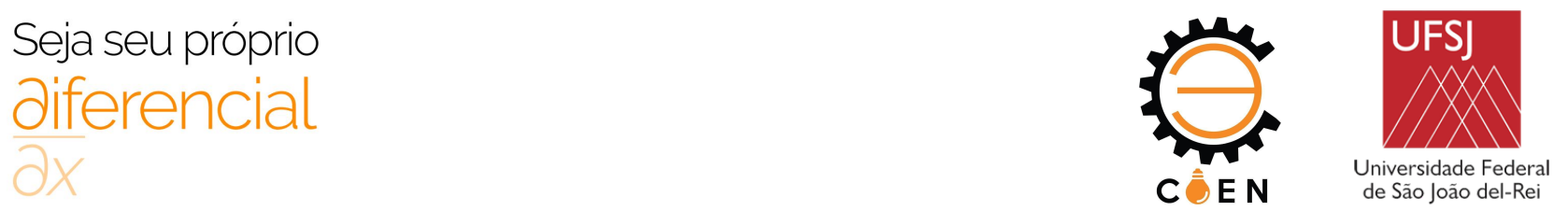

JAFFAR-BANDJEE, Mourad; CASAS, Jerome; KRIJNEN, Gijs. Additive manufacturing: state of the art and potential for insect science. Current opinion in insect science, 2018.

KOESTER, L; TAHERI, H.; BOND, L.J.; BERNARD, D.; GRAY, J. Additive manufacturing metrology: State of the art and needs assessment. In: AIP Conference Proceedings. AIP Publishing, 2016. p. 130001.

KYLE, Stuart; JESSOP Zita M.; AL-SABAH Ayesha; WHITAKER Iain S. Printability'of Candidate Biomaterials for Extrusion Based 3D Printing: State-of-the-Art. Advanced healthcare materials, v. 6, n. 16, p. 1700264, 2017.

LEVY, Gideon N.; SCHINDEL, Ralf; KRUTH, Jean-Pierre. Rapid manufacturing and rapid tooling with layer manufacturing (LM) technologies, state of the art and future perspectives. CIRP annals, v. 52, n. 2, p. 589-609, 2003.

MA, GuoWei; WANG, Li; JU, Yang. State-of-the-art of 3D printing technology of cementitious material-An emerging technique for construction. Science China Technological Sciences, v. 61, n. 4, p. 475-495, 2018.

MANUFACTURING. Google Trends, 2019. Disponível em: $<$ https://trends.google.com/trends/explore?date=all\&q=manufacturing $>$. Acesso em: 29 de junho de 2019.

NIAKI, Mojtaba Khorram; TORABI, S. Ali; NONINO, Fabio. Why Manufacturers Adopt Additive Manufacturing Technologies: The Role of Sustainability. Journal of Cleaner Production, 2019.

PEARCE, Joshua M; BLAIS, CM; LACIAK, KJ; ANDREWS, R. 3-D printing of open source appropriate technologies for self-directed sustainable development. Journal of sustainable development, v. 3, n. 4, p. 17, 2010.

RAPID Prototyping. Google Trends, 2019. Disponível em: $<$ https://trends.google.com/trends/explore?date=all\&q=rapid+prototyping $>$. Acesso em: 29 de junho de 2019.

SAMPAIO, RF \& MANCINI, MC. Estudos de revisão sistemática: um guia para síntese criteriosa da evidência científica. 2007.

ROSOCHOWSKI, A. \& MATUSZAK, A. Rapid tooling: the state of the art. Journal of materials processing technology, v. 106, n. 1-3, p. 191-198, 2000.

SACHS, E. M., HAGGERTY, J. S., CIMA, M. J., \& WILliAMS, P. A.Three-dimensional printing techniques. U.S. Patent n. 5204055, 20 abr. 1993.

SCHOFFER, Filemon. How expiring patents are ushering in the next generation of 3D printing. TechCrunch, 15 de maio de 2016. Disponível em: $<$ https://techcrunch.com/2016/05/15/how-expiring-patents-are-ushering-in-the-next-generation-of-3 d-printing/>. Acesso em: 29 de junho de 2019.

SEARS, James W. Direct laser powder deposition-'State of the Art'. Knolls Atomic Power Lab., Niskayuna, NY (US), 1999.

STEWART, Duncan. 3D printing growth accelerates again: TMT Predictions 2019. Deloitte, 2019.

Disponível

em:

$<$ https://www2.deloitte.com/insights/us/en/industry/technology/technology-media-and-telecom-pre dictions/3d-printing-market.html>. Acesso em: 28 de junho de 2019. 


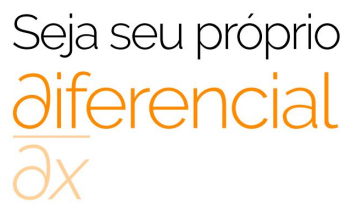

SUN, Jie; PENG, Zhuo; ZHOU, Weibiao ; FUH, Jerry Y.H.; HONG, Geok Soon; CHIU, Annette. A review on 3D printing for customized food fabrication. Procedia Manufacturing, v. 1, p. 308-319, 2015.

WONG, Kaufui V. \& HERNANDEZ, Aldo. A review of additive manufacturing. ISRN Mechanical Engineering, v. 2012, 2012.

\section{ADDITIVE MANUFACTURING: A SYSTEMATIC REVIEW}

Pedro Coelho Silva ${ }^{(1)}$ (ocoelhopedro@gmail.com), Rafael Souza Santandrea ${ }^{(2)}$ (r.santandrea97@gmail.com), Marcos Vinício Antônio Xavier ${ }^{(3)}$ (marcosxavier_1992@hotmail.com), Lincoln Cardoso Brandão ${ }^{(4)}$ (lincoln@ufsj.edu.br)

\footnotetext{
(1) Universidade Federal de São João del-Rei (UFSJ) - Engenharia Mecânica - Praça Frei Orlando, nº 170 - Centro - São João del-Rei-MG

(2) Universidade Federal de São João del-Rei (UFSJ) - Engenharia Mecânica - Praça Frei Orlando, nº 170 - Centro - São João del-Rei-MG

${ }^{(3)}$ Universidade Federal de São João del-Rei (UFSJ) - Engenharia Mecânica - Praça Frei Orlando, nº 170 - Centro - São João del-Rei-MG

(4) Universidade Federal de São João del-Rei (UFSJ) - Engenharia Mecânica - Praça Frei Orlando, nº 170 - Centro - São João del-Rei-MG
}

ABSTRACT: Additive manufacturing is realised as an technology with tendency to be one of the leading manufacturing methodologies for the future, even existing for more than 30 years presents an potential growth. Characterized by the mechanisms of fabrication by layer overlap, through various bonding mechanisms, additive manufacturing allows to construct geometries impossible or very difficult to obtain in other forms of fabrication. In the last decade there has been an increase in equipment accuracy, reduced costs, greater variety of applicable materials and possible uses. Using a methodology of systematic review, analysing articles that follow the same kind of analysis, the present investigation aims to identify which factors allowed its recent popularization and which made it difficult to implement it previously. The extinction of the main patents was identified as one of the main factors, allowing researches without the need for licensing, and with those, the beginning of a new class of equipment, with lower cost, extending the possible reach on its target market.

KEYWORDS: additive manufacturing, $3 d$ printing, state-of-the-art, systematic review. 